\title{
Efficient Localization System for Mobile Agents Using Active Beacons
}

\author{
Suyoung Shin*, Jongsuk Choi** and Mignong Park* \\ *Dept. of Electrical and Eletronics Eng., Yonsei University, \\ **Intelligence Robotics Research Center in KIST(Korea Institute of Science and Technology), \\ \{manofsun,cjs\}@kist.re.kr, mignpark@yonsei.ac.kr
}

\begin{abstract}
In this paper we propose an efficient method of localization for mobile agents by using the active beacon. Active beacons include the ultrasonic sensor, RF module and 8bit microprocessor. Existent systems have the constraint that one beacon cannot cover wide area since ultrasonic sensors have limits in the angle of signal (beam-angle) on which their signal strength depends. We make the beacons active by adding pan-tilt mechanism. In this way, we can use the strongest signal of the ultrasonic waves. As a result, the accuracy of active beacon system has been improved. And it is possible to measure local positions in wider area with this system than with existent beacon systems. In addition, this system is inexpensive because it can decrease the number of beacons by about the half of the existent system.
\end{abstract}

Keywords: active beacon, mobile agent, indoor localization, time of flight, ultrasonic, pan-tilt

\section{Introduction}

Recently, The indoor localization problem for the specific mobile agents, such as industrial robots of the factory, home service robots, the toy robots and motion recognition systems, have been getting highly interested. And many researches about the topic have been taken through various kinds of method. For example, there are dead-reckoning, vision method and beacon (ultrasonic sensor) method, and so on.

Dead-reckoning may be the simplest localization method [1] which is, however, fraught with many factors of error such as slippage and backlash. And vision system can be only one solution for a new environment with no prior information. But it is expensive since it is required to make high computation possible for image-processing [2].

Beacon method has good efficiency in cost-saving, easy signal-processing and accuracy [3-4]. But several factors remains to be improved for the higher efficiency. First, the existent systems have some constraints which need the sequential shoot of the signal in order to avoid interferences. Second, the area that one beacon can cover is not wide since ultrasonic sensors have constraints in the beam-angle on which their signal strength depend [5-6]. Consequently, the area in which we can perform the valid localization with one section of beacons (3 beacons) is strongly limited. Third, in case of multiple mobile agents, the efficiency of localization for fast agents would be relatively decreased if the systems' sampling time is fixed to all the agents.

In this paper, we propose two methods to solve the problems of the existient systems. First, making beacons active by adding pan-tilt mechanism, we are to solve the first two problems. As mentioned above, the ultrasonic sensors have the limited beam-angle. However, if we use the active mechanism in the multi-agents' localization, we can rotate one beacon toward some mobile agent while the other beacon toward other agent according to the judgement of RF beacon (beacon with Radio-Frequency module installed) and can transmit the ultrasonic signals with no interference. In this way, we can transmit the ultrasonic signals to the multiple mobile objects simultaneously. Therefore, wider area can be covered with the same number of beacons in the proposed localization. Second, RF beacon receives the data about each agent's velocity as well as position via RF communication from the agents. Also, the RF beacon gives a priority - how often to make localization which is based on the velocity of the mobile agents: it gives higher priority to faster object. Hence, the sampling time is changed efficiently corresponding to the velocities of mobile agents.

This paper decribes Structure of system and basic concepts (section 2), Inverse kinematics for active beacon (section 3), Dimensional localization using Newton method (section 4), Experimental results (section 5) and Conclusion(section 6).

\section{Structure of system and basic concepts}

\subsection{Basic concept of system}

Figure 1 shows the usable area with the existent beacon method. Basically, our active beacon method has a similar structure with the existent beacon system except some following aspects. 


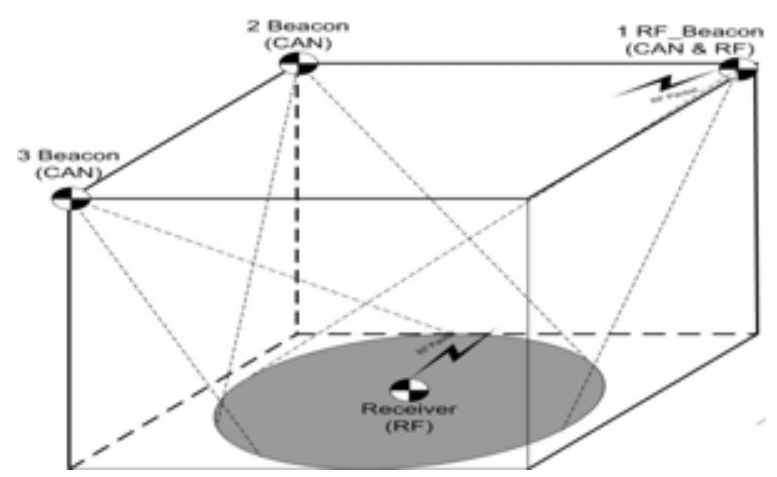

Figure 1. Usable area with fixed angle

There is timing synchronization with radio frequency communication between the RF beacon and receiver for measuring TOF (Time-Of-Flight). In this case, radio frequency communication is only used to fire beacons on existent beacon system. But it furnishes the RF beacon with some information of receiver such as velocity and position of mobile agents. RF beacon gives a priority based on the velocities of mobile agents and sends transmissionpermission-packet to them through the RF module. And it transmits the ultrasonic signal which is necessary for a localization of mobile agents by the priority. If we use this method, sampling time is changed by each velocity of mobile agents. Therefore, the density of localization for mobile agents is regularly maintained. Also RF beacon and beacons turn to the mobile agents by computing inverse kinematics before they transmit ultrasonic signal.

When each beacon completes transmission of ultrasonic signal, the receiver which is installed to each mobile agent calculates distance from the corresponding beacon. Then, the mobile agents compute local position by Newton Method.

Also, each RF beacon sends the information of neighboring section to mobile agents at boundary of section resulting in that mobile agents can continually obtain a local position even when moving section to section.

\section{2 structure of hardware}

- Receiver, RF beacon and Beacon (Figure 2):

Most electronic elements are commonly used for receivers, RF beacons and beacons. All modules include 8bit microprocessor with CAN controller. Furthermore, receivers include tone decoders for ultrasonic signal's detection. And radio frequency modules of $447 \mathrm{MHz}$ are included in only both receivers and RF beacons, and ultrasonic transmitters are included in only both RF beacons and beacons.

Receivers are connected with mobile agents through CAN or RS-232 while RF beacons are connected with beacons through CAN. Major function of beacons is firing the ultrasonic signal when they receive a command of firing from RF beacon.

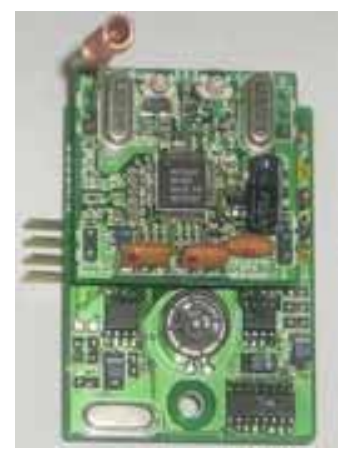

Figure 2. Receiver, RF beacon and Beacon

- Pan-tilt mechanism:

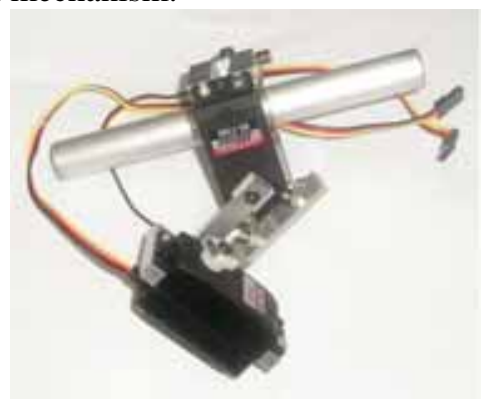

Figure 3. Pan-Tilt Mechanism

Figure 3 shows pan-tilt mechanism, having two degree of freedom, to which RF beacons and beacons are attached. Hence, RF beacons and beacons are possible to target on mobile agents located at any position of current area. Beacons are attached to the indoor space with free configuration. But RF beacons must know the attached position of beacons and themselves in section because they must furnish the information of positions to receiver. Table 1 shows configuration of hardware for each module. And figure 4 shows the usable area which is used the active beacon.

\begin{tabular}{|c|c|c|c|}
\hline $\begin{array}{c}\text { RF } \\
\text { module }\end{array}$ & Receiver & RF beacon & Beacon \\
\hline $\begin{array}{c}\text { CAN } \\
\text { Controller }\end{array}$ & $\mathrm{X}$ & $\mathrm{O}$ & $\mathrm{C}$ \\
\hline $\begin{array}{c}\text { US } \\
\text { transmitter }\end{array}$ & $\mathrm{X}$ & $\mathrm{O}$ & $\mathrm{O}$ \\
\hline $\begin{array}{c}\text { US } \\
\text { receiver }\end{array}$ & $\mathrm{O}$ & $\mathrm{X}$ & $\mathrm{X}$ \\
\hline Pan-Tilt & $\mathrm{X}$ & $\mathrm{O}$ & $\mathrm{O}$ \\
\hline
\end{tabular}

Table 1. Configuration of hardware 


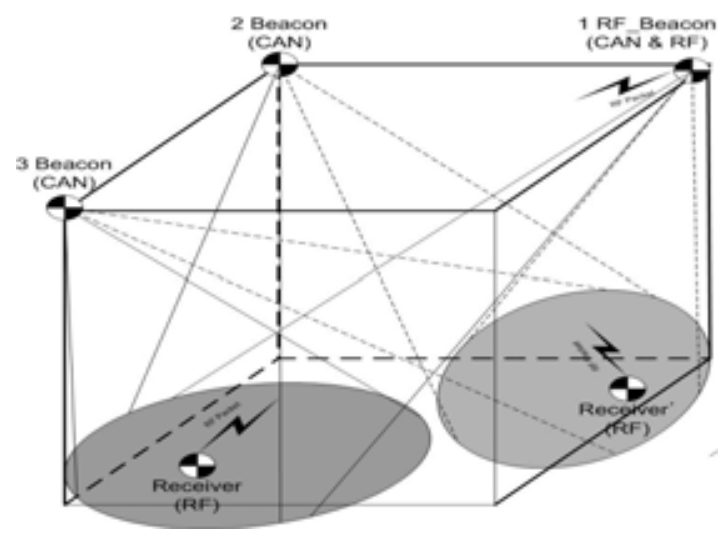

Figure 4. Usable area with active beacon

3. Inverse kinematics for active beacon

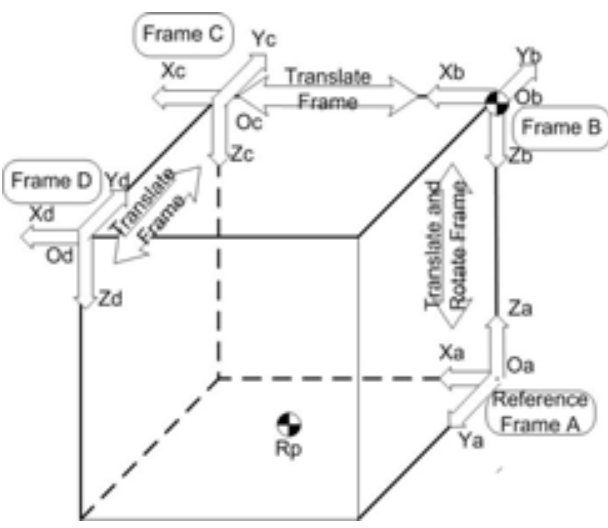

Figure 5. Coordinate frame for kinematics

Figure 5 shows translation and rotation from reference frame A to frames $B, C$ and $D$ of each $R F$ beacon and beacons. Rotations from reference frame fix on 180 degree for $\mathrm{X}$ axis, because it can reduce the amount of computation.

For example, Frame $\mathrm{B}$ translates as $O_{b}\left(x_{O b}, y_{O b}, z_{O b}\right)$ and rotates as $\left(180^{\circ}, 0^{\circ}, 0^{\circ}\right)$ from frame $\mathrm{A}$. In this place, $O_{b}$ is the central coordinate of frame $\mathrm{B}$ and position of beacons too. So ${ }^{A} R_{p}\left(x_{A}, y_{A}, z_{A}\right)$ that is receiver's position in frame $\mathrm{A}$ is expressed ${ }^{B} R_{p}\left(x_{B}, y_{B}, z_{B}\right)$ that is receiver's position in frame $B$ such as Eq. (1)

$$
\begin{gathered}
{ }^{A} R_{p}={ }^{A} T_{B}{ }^{B} R_{p} \\
{ }^{B} R_{p}={ }^{A} R_{B}^{-1}{ }^{A} R_{B}
\end{gathered}
$$

${ }^{A} T_{B}$ is homogeneous transformation matrix from frame A to frame B. So, we obtain the solution of Eq.(1)

$$
{ }^{B} R_{p}=\left[\begin{array}{c}
x_{p}-x_{O b} \\
-y_{p}-y_{O b} \\
-z_{p}-z_{O b} \\
1
\end{array}\right]
$$

We are able to calculate angles of joint1 and joint 2 using this solution. When $\theta_{1}$ is angle of joint 1 and $\theta_{2}$ is angle of joint 2, we obtain the solution by $\mathrm{Eq},(3)$

$$
\begin{gathered}
\theta_{1}=\tan ^{-1}\left(\frac{-y_{p}-y_{O b}}{x_{p}-x_{O b}}\right) \\
\theta_{2}=\tan ^{-1}\left(\frac{\sqrt{\left(x_{p}-x_{O b}\right)^{2}+\left(-y_{p}-y_{O b}\right)^{2}}}{-z_{p}-z_{O b}}\right)
\end{gathered}
$$

Figure 6 shows joints of pan-tilt mechanism.

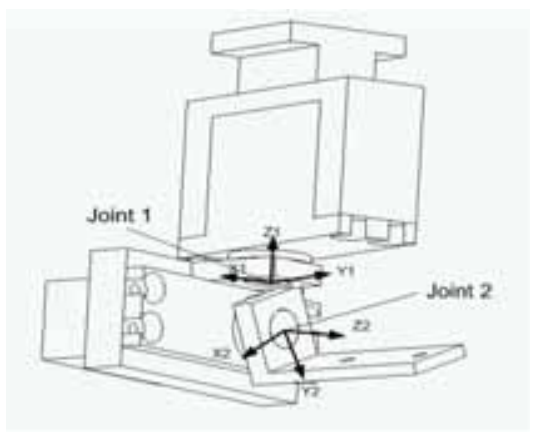

Figure 6. Coordinate Frame in Pan-tilt

\section{3 dimensional localization using Newton method}

First of all, receiver measures the distances, $r_{\mathrm{i}}$ from at least three beacons including RF beacon. Figure 7 shows method of localization.

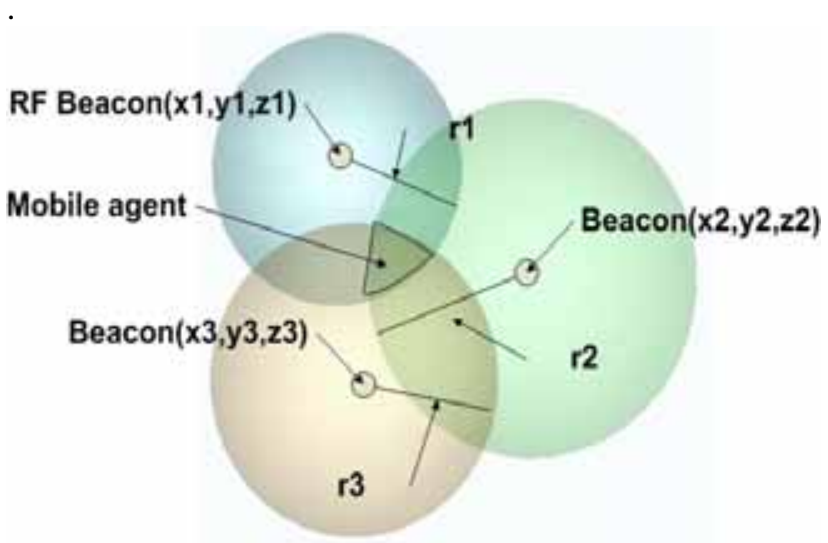

Figure 7. Concept of Localization 
Define a position vector as

$$
\mathbf{x} \triangleq\left[\begin{array}{lll}
x & y & z
\end{array}\right]^{T}
$$

Given the beacons' positions, $\left\{\mathbf{x}_{1}, \ldots, \mathbf{x}_{n}\right\}$, equations w.r.t receiver's position $\mathbf{x}$ are expressed as

$$
\left\|\mathbf{x}-\mathbf{x}_{i}\right\|^{2}=r_{i}^{2}, \quad i=1, \cdots, n
$$

If we define $F(\mathbf{x})$ as

$$
F(\mathbf{x}) \triangleq\left[f_{1} \cdots f_{n}\right]^{T}, \quad f_{i}=\left\|\mathbf{x}-\mathbf{x}_{i}\right\|^{2}-r_{i}^{2}
$$

then localization problem is equivalent to solving the following equations

$$
F(\mathbf{x})=\left[\begin{array}{lll}
0 & \cdots & 0
\end{array}\right]^{T}
$$

Since, given a $\mathbf{x}^{(k)}$ and its neighborhood $\mathbf{x}^{(k+1)}$, the $F\left(\mathbf{x}^{(k+1)}\right)$ can be approximated by Newton method like $F\left(\mathbf{x}^{(k+1)}\right) \cong F\left(\mathbf{x}^{(k)}\right)+F^{\prime}\left(\mathbf{x}^{(k)}\right)\left[\mathbf{x}^{(k+1)}-\mathbf{x}^{(k)}\right]$

where

$$
F^{\prime}\left(\mathbf{x}^{(k)}\right)=\left[\begin{array}{ccc}
\frac{\partial f_{1}}{\partial x} & \frac{\partial f_{1}}{\partial y} & \frac{\partial f_{1}}{\partial z} \\
\vdots & \\
\frac{\partial f_{n}}{\partial x} & \frac{\partial f_{n}}{\partial y} & \frac{\partial f_{n}}{\partial z}
\end{array}\right]
$$

Then, the solution is computed by iteration with a proper tolerance like

$$
\mathbf{x}^{(k+1)}=\mathbf{x}^{(k)}-\left(F^{\prime}\left(\mathbf{x}^{(k)}\right)\right)^{*} F\left(\mathbf{x}^{(k)}\right)
$$

where the $A^{*}$ means the pseudo inverse of $A$.

\section{Experimental results}

\subsection{Experimental environment}

Next following figures show the simple top view of experimental space and picture of real experimental space. Active beacon rails are established on a ceiling for the flexibility in beacon's position. Also they are efficiently mounted for attaching pan-tilt mechanism. Red circles in the picture describe the active beacons established on the ceiling

\begin{tabular}{|c|c|c|c|c|}
\hline \multicolumn{5}{|c|}{ Points of Experiment } \\
\hline 1 & 2 & 3 & 4 & 5 \\
\hline$(500$, & $(500$, & $(2500$, & $(2500$, & $(1500$, \\
2500, & 500, & 500, & 2500, & 1500, \\
$500)$ & $500)$ & $500)$ & $500)$ & $500)$ \\
\hline
\end{tabular}

Table 2. Coordinate in Reference frame (fixed angle)

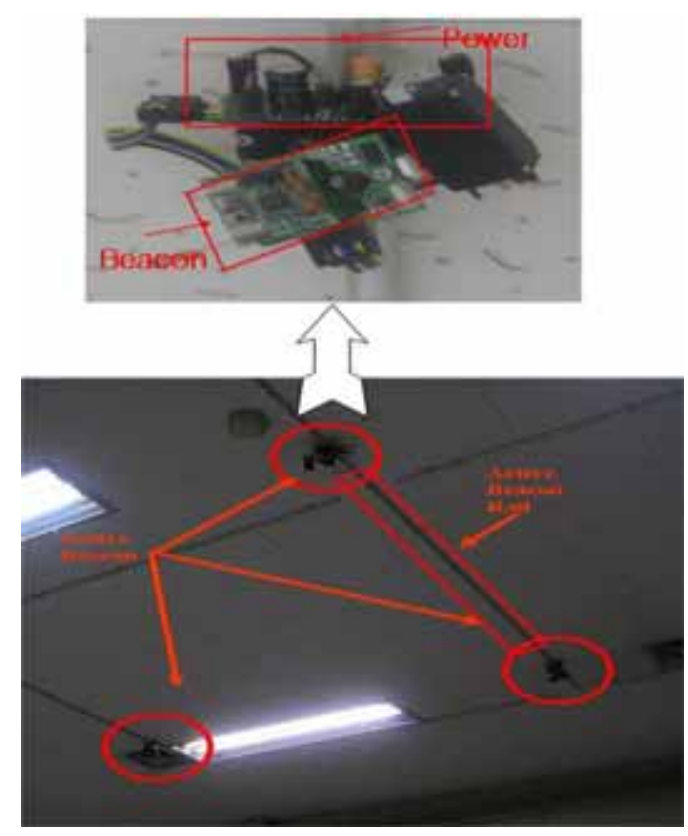

Figure 8. Active beacon and Beacon set

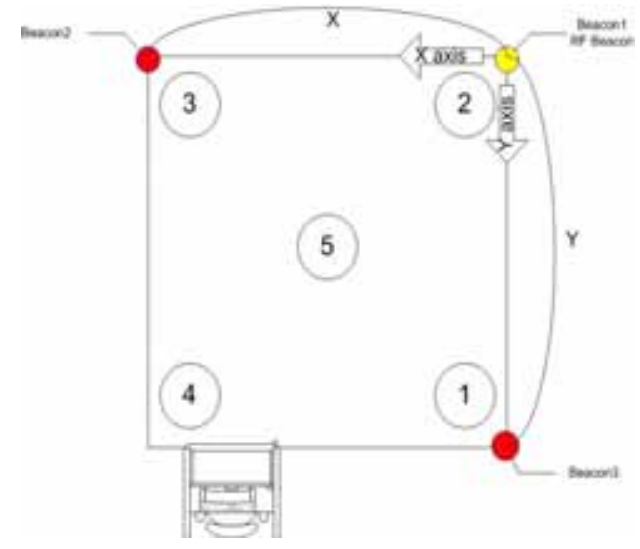

Figure 9. Simple top view and test position

\subsection{Experiment with fixed angle}

We obtain the results such as table 3 and figure 10 which are experimented in $3000 \mathrm{~mm}$ of $\mathrm{X}$ length and $3000 \mathrm{~mm}$ of $\mathrm{Y}$ length (Through all the results, the basic unit is millimeter). Circles of 1 to 5 in Figure 8 show points of experiment and its coordinates are shown in Table 1. Experiments have been performed 10 times per each point of experiment. Table 3 shows the successful receiving rate of ultrasonic signal on each point. Also Figure 10 shows local position's error of each point.

\begin{tabular}{|c|c|c|c|c|c|}
\hline \multirow{2}{*}{ BeaconID } & \multicolumn{6}{|c|}{$\begin{array}{c}\text { Successful receiving rate on each point } \\
\text { (unit: \%) }\end{array}$} \\
\cline { 2 - 6 } & 1 & 2 & 3 & 4 & 5 \\
\hline 1 & 100 & 100 & 100 & 100 & 100 \\
\hline 2 & 80 & 100 & 100 & 40 & 100 \\
\hline 3 & 100 & 100 & 100 & 100 & 90 \\
\hline
\end{tabular}

Table 3. Successful receiving rate on each point (fixed angle) 
We can find out the bad result at point 4, because all of beacons are targeted to point 5 . As mentioned above, ultrasonic signal which reached point 4 should be weak since ultrasonic sensor has the limited beam angle. On the other hand, weak ultrasonic signal gives a bad effect on the accuracy of localization. In this experiment, ultrasonic sensors have firing time of 450us at least which is required for the tone decoder of receiver. The weak ultrasonic signal is detected latter than the strong signal resulting in that it causes large, values of error as shown in Figure 10.

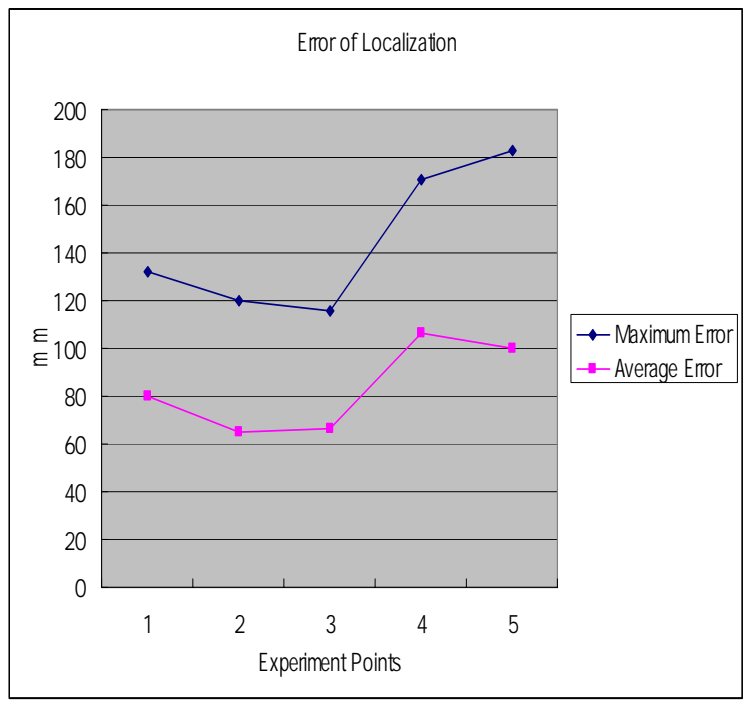

Figure 10. Maximal and Average Error with fixed angle

\subsection{Experiment with variable angle(active beacons)}

Experiments of active beacon system are executed in a space whose size is $4000 \mathrm{~mm}$ by $5700 \mathrm{~mm}$ and table 4 and figure 11 show the results of experiments.

\begin{tabular}{|c|c|c|c|c|c|}
\hline \multirow{2}{*}{$\begin{array}{c}\text { beacon } \\
\text { ID }\end{array}$} & \multicolumn{5}{|c|}{$\begin{array}{c}\text { Successful receiving rate on each point } \\
\text { (unit: \%) }\end{array}$} \\
\cline { 2 - 6 } & 1 & 2 & 3 & 4 & 5 \\
\hline 1 & 100 & 100 & 100 & 100 & 100 \\
\hline 2 & 100 & 100 & 100 & 100 & 100 \\
\hline 3 & 100 & 100 & 100 & 100 & 90 \\
\hline
\end{tabular}

Table 4. Successful receiving rate on each point (active beacons)

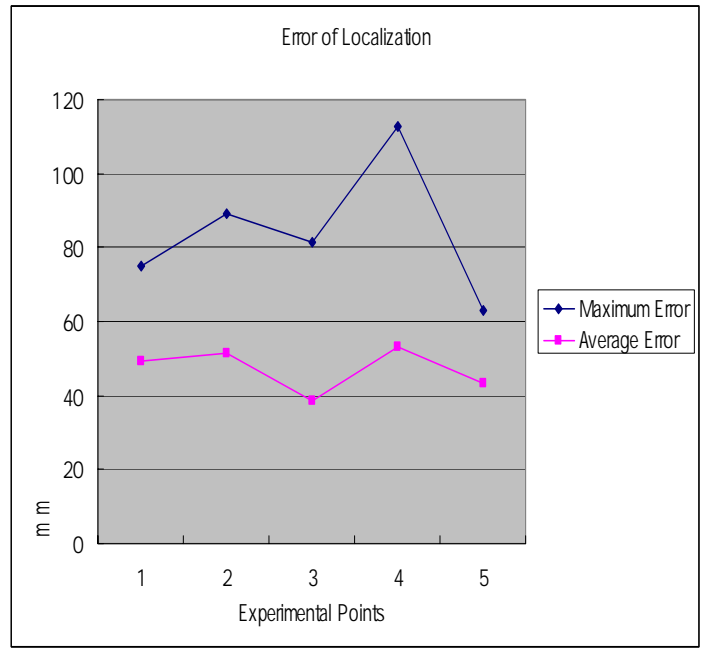

Figure 11. Maximal and Average Error with variable angle (active beacons)

As we expected, both the successful receiving rate and the accuracy in the active beacon system are better: maximal and average errors are reduced by a half and the successful receiving rates remains high even at the point 4 .

\section{Conclusion}

Beacon system which is one of localization's methods is very good for cost-saving, accurate localization. We have performed experiments with the existent method which has a fixed angle. And we can find out the problem of the existent beacon method. As mentioned above, existent researches of beacon method are not efficient. Hence, we propose the active beacon system in this paper that attaches beacon to pan-tilt mechanism. As shown in the results, active beacon system is better than the existent beacon system in two factors - both the successful receiving rate and the accuracy of localization. Naturally, this system is not perfect up to now. Average error is over the $30 \mathrm{~mm}$ and maximal error over the $60 \mathrm{~mm}$.

However, we confirm the possibility for efficient localization. And the accuracy and the successful receiving rates heavily depend on the electronic circuits and software's delay. The delay by software is factor of error each distance between receivers and beacons.

We will make maximal error under 50mm.In addition and make the area which one set of beacon covers over $6 \mathrm{~m}$ by $6 \mathrm{~m}$. So we are planning to use our active beacon system in the building for the localization of mobile robots. Then, they can detect their absolute positions by themselves, and also can have the flexibility on their free movements.

\section{REFERENCES}


[1] Y. Zhao, Vehicle Location and Navigation Systems, Artech House Publish, 1997.

[1] Pentland, "Machine Understanding of Human Action", Proceeding of $7^{\text {th }}$ International Forum on Frontier of Telecommunication Technology, 1995.

[3] Soo-Yeong Yi and Jae-Ho Jin, "Self-localization of a Mobile Robot Using Global Ultrasonic Sensor System", Journal of Control, Automation, and Systems Engineering, Vol. 9 No.2, pp. 145-151, 2003.

[4] Y. G. Kim, J. S. Choi, J. O. Kim, and M. Kim, I. M., "A New Localization System for Mobile Robots Using Radio Frequency and Ultrasound", 2002 International conference on Control, Automation, and Systems, pp. 148-152, 2002.

[5] Nissanka B. Priyantha, Anit Chakraborty, and Hari Balakrishnan, "The Cricket Location-Support System", $6^{\text {th }}$ ACM International Conference on Mobile Computing and Networking (ACM MOBICOM), pp 32-43, 2000.

[6] Hari Balakrishnan, Roshan Baliga, Dorothy Curtis, Michel Goraczko, Allen Miu, Nissanka B. Priyantha, Adam Smith, Ken Steele, Seth Teller, Kevin Wang, "Lessons from Developing and Deploying the Cricket Indoor Location System", MIT Computer Science and Artificial Intelligence Laboratory (CSAIL), 2003. 